ARTICLE

\title{
The immunoglobulin tail tyrosine motif upgrades memory-type BCRs by incorporating a Grb2-Btk signalling module
}

Niklas Engels ${ }^{1, \star}$, Lars M. König ${ }^{1, \star}$, Wiebke Schulze ${ }^{1}$, Daniel Radtke ${ }^{2}, K^{2}$ anika Vanshylla ${ }^{1}$, Johannes Lutz ${ }^{1}$, Thomas H. Winkler ${ }^{3}$, Lars Nitschke ${ }^{2}$ \& Jürgen Wienands ${ }^{1}$

The vigorous response of IgG-switched memory $B$ cells to recurring pathogens involves enhanced signalling from their B-cell antigen receptors (BCRs). However, the molecular signal amplification mechanisms of memory-type BCRs remained unclear. Here, we identify the immunoglobulin tail tyrosine (ITT) motif in the cytoplasmic segments of membrane-bound IgGs (mlgGs) as the principle signal amplification device of memory-type BCRs in higher vertebrates and decipher its signalling microanatomy. We show that different families of protein tyrosine kinases act upstream and downstream of the ITT. Spleen tyrosine kinase (Syk) activity is required for ITT phosphorylation followed by recruitment of the adaptor protein Grb2 into the mlgG-BCR signalosome. Grb2 in turn recruits Bruton's tyrosine kinase (Btk) to amplify BCR-induced $\mathrm{Ca}^{2}+$ mobilization. This molecular interplay of kinases and adaptors increases the antigen sensitivity of memory-type BCRs, which provides a cellintrinsic trigger mechanism for the rapid reactivation of IgG-switched memory $B$ cells on antigen recall.

\footnotetext{
${ }^{1}$ Institute of Cellular and Molecular Immunology, Georg-August-University of Göttingen, Medical Faculty, Humboldtallee 34, 37073 Göttingen, Germany. ${ }^{2}$ Chair of Genetics, Department of Biology, Friedrich-Alexander-University Erlangen-Nürnberg, Staudtstrasse 5, 91058 Erlangen, Germany. ${ }^{3}$ Hematopoiesis Unit, Department of Biology, Nikolaus-Fiebiger-Center for Molecular Medicine, Friedrich-Alexander-University Erlangen-Nürnberg, Glückstrasse 6, 91054 Erlangen, Germany. ${ }^{\star}$ These authors contributed equally to this work. Correspondence and requests for materials should be addressed to N.E. (email: nengels@gwdg.de) or to J.W. (email: jwienan@gwdg.de).
} 
H umoral immunity is based on the establishment of protective antibody titres as well as the formation of long-lived memory B cells that are poised to swiftly differentiate into antibody-producing plasma cells on antigen reencounter ${ }^{1,2}$. Memory B cells express either membrane-bound IgM (mIgM) or class-switched mIg isotypes, most notably mIgGs, as part of the B-cell antigen receptor (BCR) on their cell surface $^{3,4}$. However, mIgG-expressing memory B cells are preferentially reactivated over mIgM-expressing cells on antigen recall $^{4,5}$. Recent evidence indicated that BCR-intrinsic signal amplification of mIgG-containing BCRs is involved in this process $^{6,7}$.

The minimal BCR unit consists of an mIg molecule that noncovalently associates at a 1:1 stoichiometry with a heterodimer of mIg-associated $\alpha$ and $\beta$ transmembrane proteins $(\operatorname{Ig} \alpha / \beta$, $\mathrm{CD} 79 \mathrm{a} / \mathrm{b})^{8,9}$. Ig $\alpha$ and $\operatorname{Ig} \beta$ each contain one copy of the immunoreceptor tyrosine-based activation motif (ITAM) in their cytoplasmic domains that serves to recruit and activate cytosolic protein tyrosine kinases (PTKs) of the Src and
Syk/ZAP70 families ${ }^{10,11}$. In addition, Ig $\alpha$ contains an evolutionarily conserved non-ITAM tyrosine residue, Y204, that on phosphorylation recruits the central B-cell adaptor protein SLP65 (BLNK) via its Src homology (SH) 2 domain ${ }^{12-14}$. Phosphorylation of SLP65 by activated Syk allows the formation of a multimolecular protein complex consisting of the key enzymes for BCR-induced $\mathrm{Ca}^{2+}$ mobilization, the PTK Bruton's tyrosine kinase (Btk) and phospholipase C- $\gamma 2$ (PLC- $\gamma 2$ ), both of which bind to phospho-SLP65 via their $\mathrm{SH} 2$ domains ${ }^{15,16}$. The formation of this complex is critical for activation of PLC- $\gamma 2$ by Btk and subsequent generation of the second messengers diacylglycerol and inositol-1,4,5-trisphosphate (IP3) by cleavage of the membrane lipid phosphatidylinositol-4,5-bisphosphate. IP3 directly activates $\mathrm{Ca}^{2}+$ channels in the endoplasmic reticulum (ER) and plasma membranes and thus controls the intensity and duration of BCR-induced $\mathrm{Ca}^{2+}$ mobilization ${ }^{17-19}$.

In contrast to $\mathrm{mIgM}$ and $\mathrm{mIgD}$, mIgGs contain cytoplasmic domains of considerable length. Experiments using genetically modified mice demonstrated that the cytoplasmic tail of mIgG1 a
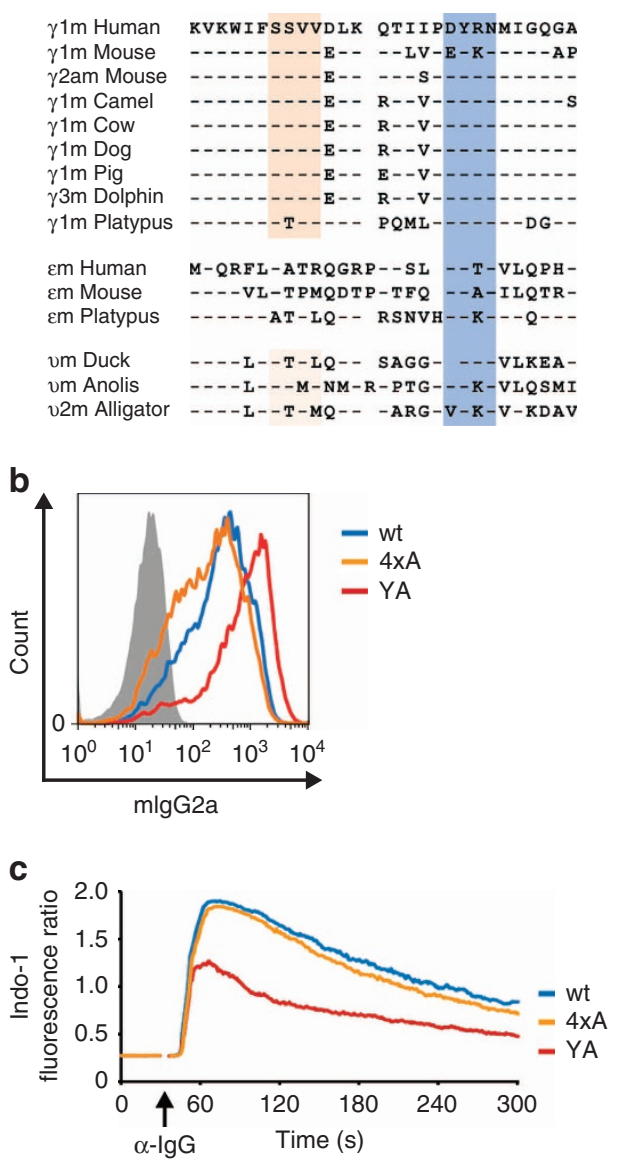

d

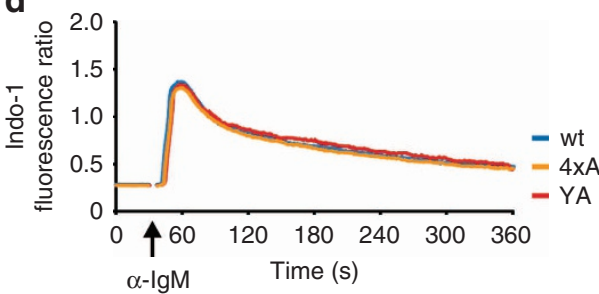

e
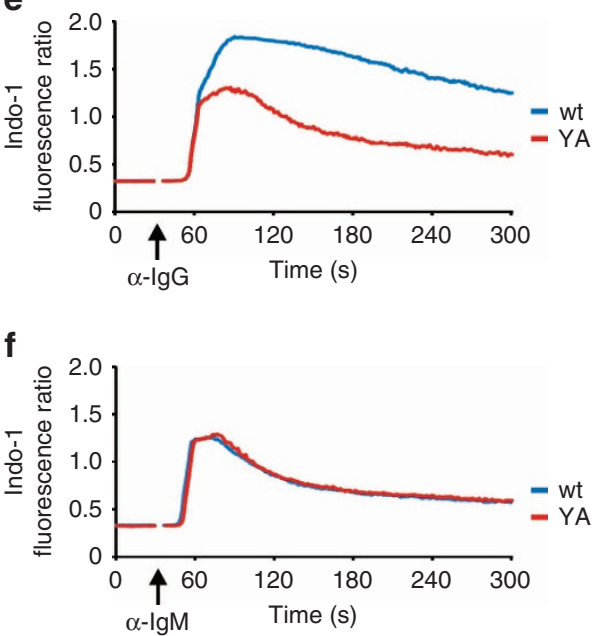

9

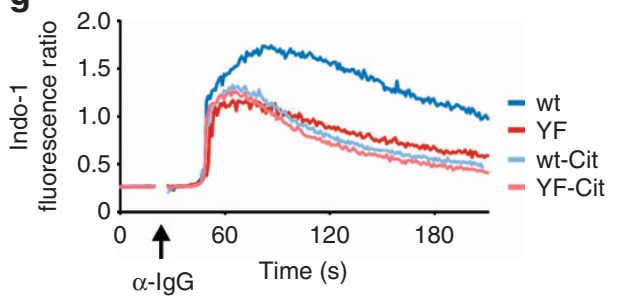

Figure 1 | The ITT is the principal signal amplification device of mlgG-containing BCRs. (a) Amino-acid sequence alignment (single-letter code for amino acids) of cytoplasmic tails of $\gamma \mathrm{m}(\mathrm{mlgG}), \varepsilon m(\mathrm{mlgE})$ or $\mathrm{vm}(\mathrm{mlgY})$ immunoglobulin heavy chains of the indicated species. The putative PDZ domain binding SSVV motif is highlighted in orange, the ITT motif in blue. (b) Surface expression of the indicated mlgG2a-BCR variants on DG75 B cells. (c,d) Ca ${ }^{2+}$ mobilization kinetics on stimulation with $\mathrm{F}\left(\mathrm{ab}^{\prime}\right)_{2}$ fragments to $\operatorname{lgG}\left(20 \mu \mathrm{gl}^{-1}\right)$ (c) and $\operatorname{lgM}\left(20 \mu \mathrm{gl}^{-1}\right)$ (d) were analysed in the presence of $1 \mathrm{mM}^{-}$ extracellular $\mathrm{CaCl}_{2}$. (e,f) DG75 cells expressing chimeric mlgG/Y molecules were analysed as before. (g) C-terminally Citrine-tagged mlgG2a variants (wt, light blue line and ITT-mutant (YF) light red line) were expressed in DG75 cells, and anti-lgG-induced Ca ${ }^{2+}$ mobilization kinetics were analysed as before. Non-tagged $\gamma 2$ am versions (dark blue and dark red lines) served as controls. Data are representative of at least three independent experiments. 
a

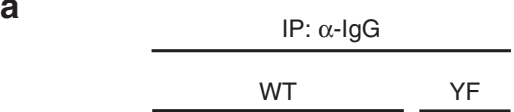

Inhibitor: $-\beta^{\beta^{1}} \beta^{2} \beta^{21}--$

$\alpha-\lg G(\min ): \quad 0 \quad 5 \quad 5 \quad 5 \quad 5 \quad 0 \quad 5$

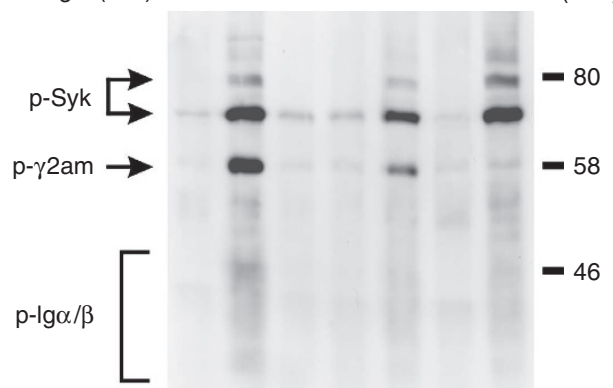

$\frac{\text { p- } \gamma 2 \mathrm{am}}{\gamma 2 \mathrm{am}}: \quad 0.01 \quad 1 \quad \begin{array}{cc}0.010 .020 .050 .020 .03 \\ \alpha-p-T y r\end{array}$
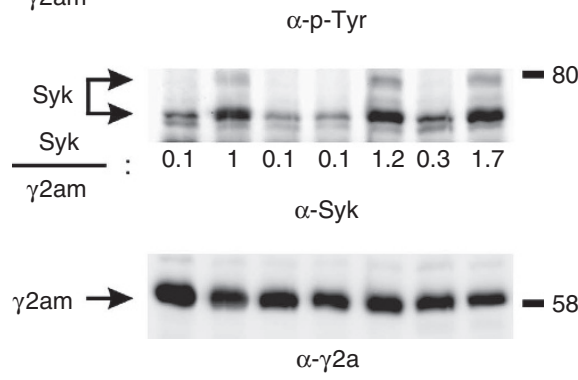

b
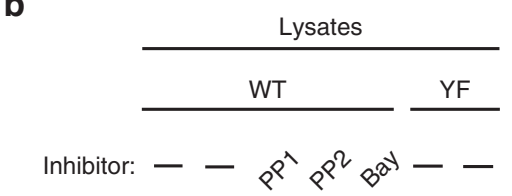

$\alpha-\lg G(\min ): \quad \begin{array}{lllllll}0 & 5 & 5 & 5 & 5 & 0 & 5\end{array}$

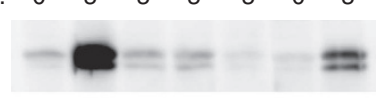

$\alpha-p-S L P 65$

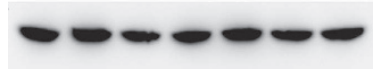

$\alpha-\beta$-Actin

$\begin{array}{lllllll}1 & 2 & 3 & 4 & 5 & 6 & 7\end{array}$

$\begin{array}{lllllll}1 & 2 & 3 & 4 & 5 & 6 & 7\end{array}$

Figure 2 | The ITT is phosphorylated by BCR-bound Syk. Ramos cells expressing either wild type (WT) or ITT-mutant (YF) mlgG2a were left untreated or pre-incubated with the Src kinase inhibitors PP1 or PP2 or the Syk inhibitor BAY61-3,606 for 30 min at room temperature. Subsequently, cells were left unstimulated $(0)$ or stimulated for 5 min with polyclonal goat anti-mouse lgG antibodies, lysed and used for affinity purifications of mlgG2a-BCRs with protein A/G agarose beads (a). Purified proteins were successively analysed with antibodies to phosphotyrosine ( $\alpha$-p-Tyr, upper panel), Syk (middle panel) and $\gamma 2 \mathrm{a}$ (lower panel). Protein band intensities were determined and normalized as indicated on the left. (b) Aliquots of the same lysates used in (a) were analysed with anti-phospho-SLP65 (Y96) (upper panel) and anti- $\beta$-Actin (lower panel) as loading control. Data are representative of three independent experiments.

plays a key role in the activation and maintenance of mIgG1expressing memory $\mathrm{B}$ cells $\mathrm{s}^{20-22}$. Recently, two distinct signalamplifying peptide motifs that differ in their proposed mode of operation were identified in the cytoplasmic domains of mammalian mIgG isotypes ${ }^{6,7,23}$. An SSVV (single-letter code for amino acids) peptide motif that is found within the 10 membrane-proximal amino acids of mammalian mIgG tails but not in mIgE tails was suggested to enhance signalling by constitutively recruiting the PDZ domain-containing scaffold protein SAP97 (ref. 23). The second motif represents a tyrosine phosphorylation-dependent interaction site for $\mathrm{SH} 2$ domaincontaining effector molecules that is evolutionarily conserved in $\mathrm{mIgG}$ and $\mathrm{mIgE}$ isotypes ${ }^{6}$. On phosphorylation, this immunoglobulin tail tyrosine (ITT) motif recruits the adaptor protein growth factor receptor-bound 2 (Grb2) into the BCR signalosome to amplify antigen-induced $\mathrm{Ca}^{2}+$ mobilization and cellular proliferation. Inactivation of the ITT motif abolished enhanced mIgG-BCR signalling resulting in an mIgM-BCR-like signalling profile ${ }^{6}$.

The ubiquitous adaptor protein Grb2 has multiple roles in B cells. Initially, it was recognized as part of an inhibitory signal complex together with the adaptor protein Dok-3 that restricts BCR-induced $\mathrm{Ca}^{2}+$ mobilization in mIgM-expressing cells ${ }^{17,24-26}$. Furthermore, it may be involved in signalling of the negative regulatory coreceptor $\mathrm{CD} 22^{27}$ and chemokine receptors in germinal centre $\mathrm{B}$ cells ${ }^{28}$. However, recently we provided evidence that in mIgG-expressing cells Grb2 promotes B-cell activation by supporting the mobilization of $\mathrm{Ca}^{2+}$ on binding to the phosphorylated ITT. Yet, the microanatomy of this signalamplifying mechanism that underlies the robust reactivation of IgG-switched memory B cells remained unclear. Here, we demonstrate that the ITT motif represents the principle signal amplification device of memory-type BCRs in higher vertebrates. We find that PTKs of different families are involved in ITT-mediated signal amplification. Whereas Syk catalyses the phosphorylation of the ITT to allow for the recruitment of Grb2, Btk is involved in subsequent amplification of PLC- $\gamma$ activity and sustained $\mathrm{Ca}^{2}+$ mobilization. Collectively, these mechanisms greatly increase the antigen sensitivity of isotype-switched B cells compared with cells that solely rely on ITAM-induced activation signals, which can explain their preferential reactivation in secondary immune responses.

\section{Results}

The ITT is the main signal amplifier of memory-type BCRs. The cytoplasmic domains of $\mathrm{mIgG}$ isotypes have been reported to contain two distinct signal-amplifying peptide motifs. One comprises the amino acids $\mathrm{SSVV}^{23}$ and is evolutionarily conserved within the 10 membrane-proximal amino acids of mammalian mIgG tails but not in mIgE and only to some extent in mIgY tails of birds and reptiles (Fig. 1a). By contrast, the ITT 

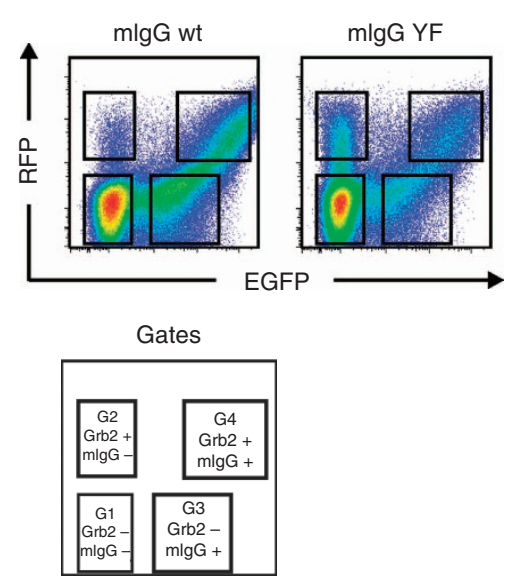

b

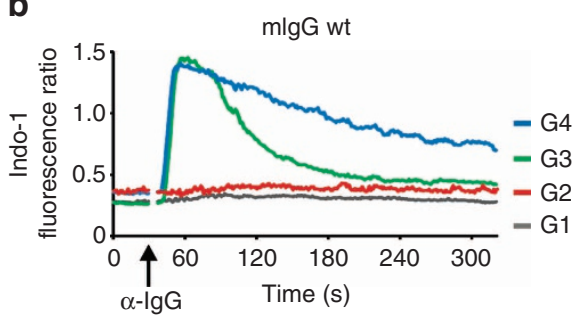

c

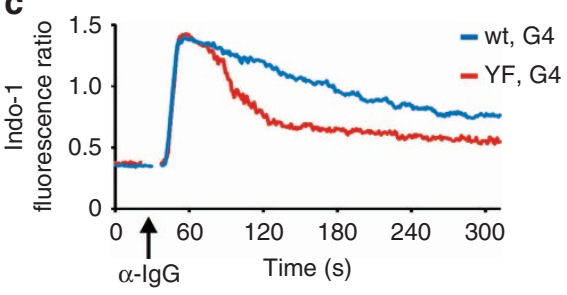

d

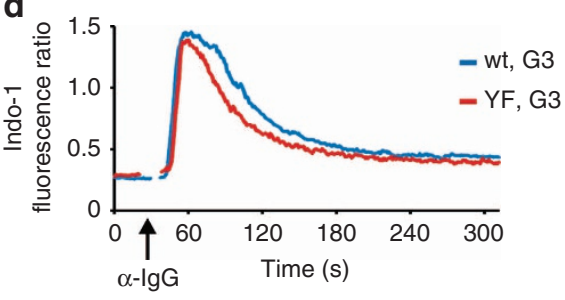

e

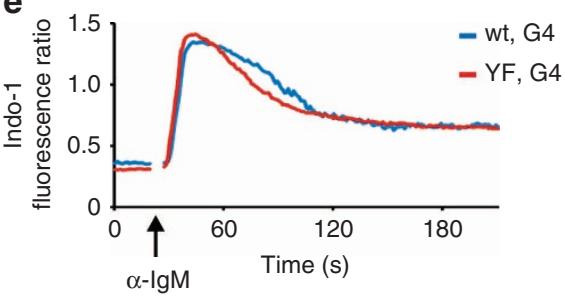

Figure 3 | ITT signalling requires expression of Grb2. (a) Splenic mouse B cells deficient for Grb2 were retrovirally transfected with expression vectors encoding mouse $\gamma 2 \mathrm{am}$ (wt or YF) together with IRES-driven EGFP and Grb2 along with IRES-driven RFP. The resulting populations in the different gates (G1-G4) are shown on the right. The cells were stimulated with $F\left(a b^{\prime}\right)_{2}$ fragments against $\operatorname{lgG}\left(\alpha-\operatorname{lgG}\right.$, indicated by an arrow, $20 \mu \mathrm{g} \mathrm{ml}^{-1}$ ) (b-d) or $\operatorname{lgM}\left(\alpha-\operatorname{lgM}, 20 \mu \mathrm{g} \mathrm{ml}{ }^{-1}\right)(\mathbf{e})$. (b) $\mathrm{Ca}^{2+}$ mobilization kinetics of cells from all gates transfected with wild-type $\gamma 2$ am. (c,d) Ca ${ }^{2+}$ mobilization kinetics of cells expressing either wild type (wt, blue curves) or YF-mutant (red curves) mlgG2a-BCRs from gate G4 (c) and G3 (d) on anti-lgG stimulation or $\mathrm{G} 4$ on anti-lgM stimulation (e). $\mathrm{Ca}^{2}+$ mobilization was recorded in the presence of $1 \mathrm{mM}$ extracellular $\mathrm{CaCl}_{2}$. Data are representative of three independent experiments.

motif is conserved not only in mIgGs but also in mIgE as well as in primordial mIgY molecules (Fig. 1a). To assess the individual contribution of the different peptide motifs for enhanced mIgGBCR signalling, we generated alanine mutants of the SSVV (4xA) and ITT (YA) motifs and compared their $\mathrm{Ca}^{2+}$ mobilization capacities with that of wild-type BCRs. Surface expression of the different $\mathrm{mIgG}-\mathrm{BCR}$ variants in the human B-cell line DG75 was similar, even though the YA variant was somewhat more abundant on the cell surface than wild type and 4xA-mutant mIgG (Fig. 1b). As shown in Fig. 1c, inactivation of the SSVV motif (orange line) had little effect on mIgG-BCR-mediated signal amplification as opposed to the robust drop caused by inactivation of the ITT (red line). As control, all cells were stimulated via their endogenous mIgM-BCRs to verify equal ITAM-mediated $\mathrm{Ca}^{2+}$ signalling capacities of the transfectants (Fig. 1d). To test whether the ITT motifs in primordial mIgY molecules of reptiles and birds are functionally conserved, we generated chimeric $\mathrm{mIgG} / \mathrm{Y}$ molecules in which the cytoplasmic domain of mouse $\gamma 2 \mathrm{am}$ was replaced with that of um from the lizard Anolis carolinensis. Stimulation of this chimeric receptor caused a very strong and sustained $\mathrm{Ca}^{2+}$ response, which was reverted to an mIgM-BCR-like response on inactivation of the Anolis ITT (Fig. 1e). As before, the integrity of the $\mathrm{Ca}^{2+}$ pathway in the transfectants was verified through stimulation of their endogenous mIgM-BCRs (Fig. 1f). These results show that the ITT is the principal signal amplification device of memory-type BCRs in higher vertebrates.

However, microscopic analyses of mIgG-molecules that were fused to a fluorescent protein tag at their $\mathrm{C}$ termini (that is, intracellular) suggested that the ITT motif plays a minor part in enhancing $\mathrm{Ca}^{2}+$ mobilization ${ }^{7}$. In search of an explanation for these conflicting results, we generated C-terminally Citrinetagged variants of $\mathrm{mIgG}$ and tested whether such intracellular fluorophore tagging of $\mathrm{mIgG}$ tails influences their signalling behaviour. Indeed, the fluorophore tag completely abrogated ITT-mediated amplification of $\mathrm{Ca}^{2+}$ mobilization (Fig. 1g), which might be caused by sterical hindrance of $\mathrm{SH} 2$ domainmediated binding to the phospho-ITT. On balance, the ITT motif, which appeared earlier in vertebrate evolution than the SSVV motif and furthermore has been conserved in $\mathrm{mIgY}, \mathrm{mIgG}$ and mIgE molecules, appears to be the major signal-boosting element of memory-type BCRs.

The ITT is phosphorylated by the BCR transducer kinase Syk. To better understand the molecular microanatomy of ITTmediated signal amplification, we addressed the question as to which kinase acts upstream of the ITT. We previously showed that efficient phosphorylation of the ITT requires its incorporation into the BCR complex ${ }^{6}$. To test which of the two BCR-bound PTKs, Src-family kinases (SFKs) or Syk, phosphorylates the ITT, we analysed its phosphorylation in mIgG-expressing human B cells that were pre-incubated with either the SFK inhibitors PP1 or PP2 or the Syk inhibitor BAY61-3606 (Fig. 2 and Supplementary Fig. 1). Treatment of the cells with either PP1 or PP2 reduced not only BCR-induced tyrosine phosphorylation of the ITT to background levels but also that of Ig $\alpha / \beta$, Syk (Fig. $2 \mathrm{a}$ upper panel, lanes 3 and 4) and SLP65 (Fig. 2b), consistent with 
a

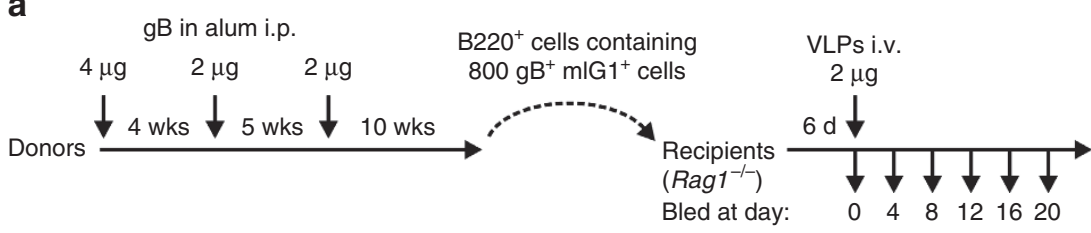

b

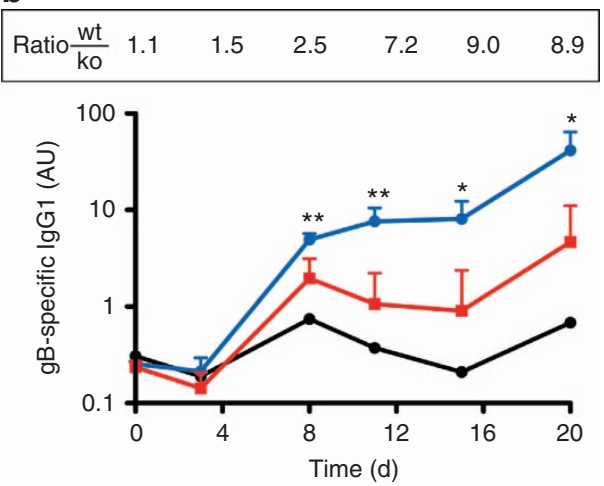

C

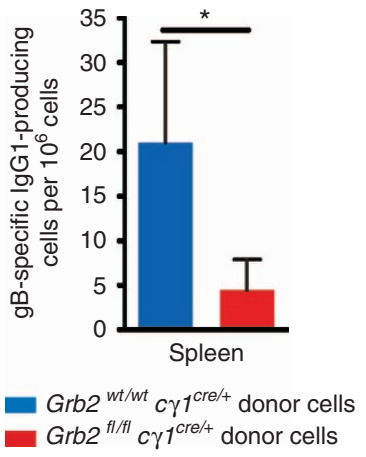

- Grb2 wt/wt $c \gamma 1^{\text {cre/t }}$ donor cells

- Grb2 ${ }^{f / / f l} c 1^{\text {cre/t }}$ donor cells

— Non-immunized donor cells

Figure 4 | T-cell-independent reactivation of IgG-switched memory B cells requires Grb2. (a) Experimental outline. Grb2 ${ }^{w t / w t} C \gamma 7^{c r e} /+(n=3)$ and $\mathrm{Grb2} 2^{f / f l} \mathrm{C} \gamma 7^{c r e /}+$ mice $(n=5)$ were repeatedly immunized with purified $\mathrm{gB}$ with aluminium hydroxide (alum) at the indicated time points. Splenic $\mathrm{B}$ cells were purified 70 days after third immunization by complement-mediated T-cell lysis and anti-CD19 magnetic bead separation. CD19-positive cells containing 800 memory B cells each (identified as B220 +, lgG1+,gB+) were transferred intravenously into Rag1 ${ }^{-/}$- recipient mice and challenged 6 days later by an intravenous injection of $2 \mu \mathrm{g}$ virus-like particles (VLPs) of human CMV in PBS. (b) gB-specific lgG1 titres were measured by ELISA. (c) ELISPOT for gB-specific IgG1-secreting cells was performed at day 43 after VLP challenge. Error bars represent mean $+\mathrm{s}$.d. of at least three analyses; Student's $t$-test was used. ${ }^{\star} P<0.05{ }^{\star \star} P<0.01$.

the common view that SFKs are the most upstream PTKs in the BCR signalling cascade. Notably, inhibition of SFKs disrupted the association between the BCR and Syk (Fig. 2a, middle panel, lanes 3 and 4), leaving open the possibility that recruitment of Syk to the BCR was required for ITT phosphorylation. By contrast, pharmacological inhibition of Syk did not interfere with its association with Ig $\alpha / \beta$ (Fig. 2a, middle panel, lane 5), yet phosphorylation of the ITT was almost completely extinguished (lane 5, upper panel). The effectiveness of Syk inhibition was demonstrated by the absence of BCR-induced SLP65 phosphorylation in the cells (Fig. 2b, lane 5). Thus, Syk appears to be the kinase that directly phosphorylates the ITT, whereas SFKs are essential for phosphorylation of $\operatorname{Ig} \alpha / \beta$ and hence activation of Syk.

The adaptor protein Grb2 is an essential ITT effector. A direct binding partner of the phosphorylated ITT is the ubiquitous adaptor protein Grb2 (ref. 6). To test whether Grb2 expression was required for ITT-mediated amplification of proximal BCR signalling events in a genetic model system, we retrovirally expressed either wild type (wt) or tyrosine to phenylalaninemutant (YF) mIgG along with EGFP in murine splenic B cells from mice with a B-cell-specific deletion of the Grb2 gene ${ }^{26}$. Measurement of mIgG-BCR-induced $\mathrm{Ca}^{2}+$ mobilization showed that the signal-amplifying effect of the ITT was abolished in Grb2-deficient B cells (Supplementary Fig. 2). Next, we doubly transduced Grb2-deficient primary B cells with wt or YF-mutant $\gamma 2 \mathrm{am}$ together with a second retroviral vector that reconstituted the cells with Grb2 along with IRES-driven expression of a red fluorescent protein (RFP). This transduction strategy gave rise to four populations of cells that could be distinguished according to the fluorescence of EGFP and/or RFP (Fig. 3a). B cells expressing wt mIgG together with Grb2 (gate G4 in Fig. 3a) showed a strong and long-lasting BCR-induced $\mathrm{Ca}^{2}+$ flux (Fig. 3b, blue curve) compared with the transient $\mathrm{Ca}^{2}+$ release in cells expressing mIgG but no Grb2 (gate G3, Fig. 3b, green curve). Cells that were EGFP-negative (gates G1 and G2) and hence did not express mIgG did not respond to mIgG-BCR stimulation (red and grey lines). Comparison of cells expressing either wt or YF-mutant mIgG-BCRs along with Grb2 from gate G4 (Fig. 3c) and those from gate G3 (Fig. 3d) showed that both the presence of a functional ITT as well as the expression of Grb2 were needed for enhanced mIgG-BCR-mediated $\mathrm{Ca}^{2+}$ signalling. Stimulation of endogenous mIgM-BCRs showed that transduced cells from G4 had the same ITAM-mediated signalling capacities (Fig. 3e). Similar results were obtained in the human B-cell line DG75 in which we inactivated the GRB2 gene by gene targeting (see Supplementary Figs 3 and 4). These genetic experiments identify the universal adaptor protein Grb2 as essential component for improved signalling of mIgG-BCRs, which is consistent with the dispensability of the putative SSVV motif in that process.

IgG-switched memory B cells require Grb2 for reactivation. Having established that the ITT of memory-type BCRs and the adaptor protein Grb2 constitute a functional duo, we tested whether Grb2 is required for the reactivation of IgG-switched memory B cells in vivo using a genetic model system. To this end, we generated mice in which the Grb2 gene gets deleted in germinal centre (GC) B cells by crossing Grb2 fl/fl mice ${ }^{26}$ with ${ }_{C} \gamma 1^{\text {cre }}$ mice ${ }^{29}$. In the resulting Grb2 $2^{f l f l} C \gamma 1^{\text {crel }+}$ animals, the expression of Grb2 is efficiently shut off in GC B cells (Supplementary Fig. 5). The mice were repeatedly immunized with purified glycoprotein $\mathrm{B}(\mathrm{gB})$ of human cytomegalovirus (hCMV) to induce formation of an antigen-specific 
a
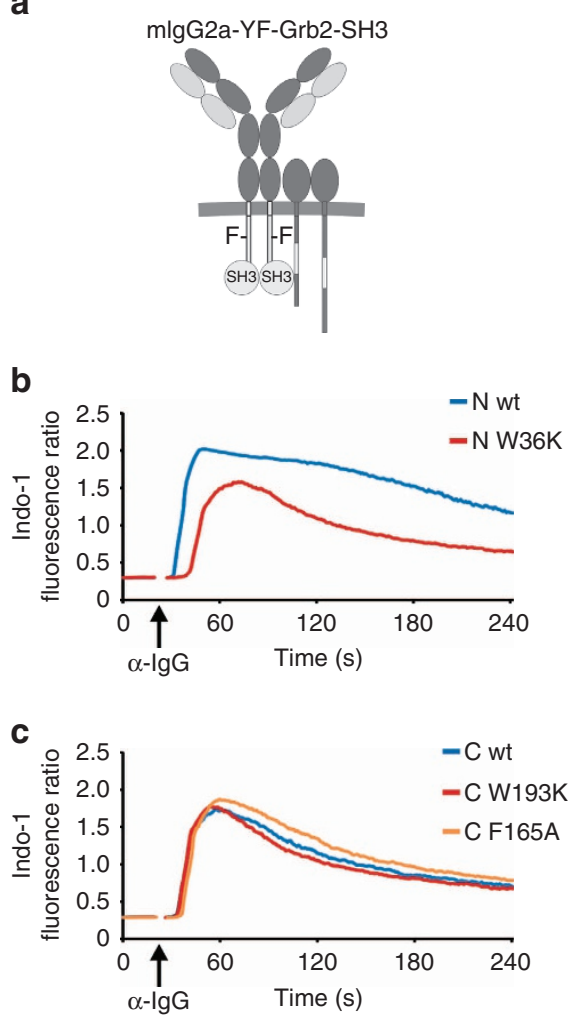

d

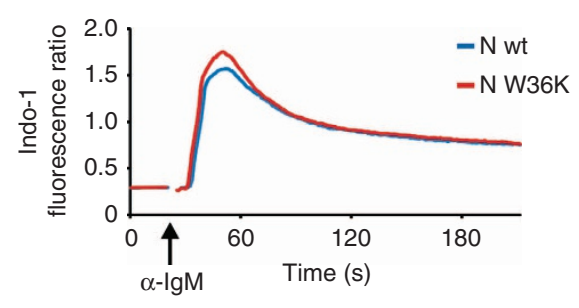

e

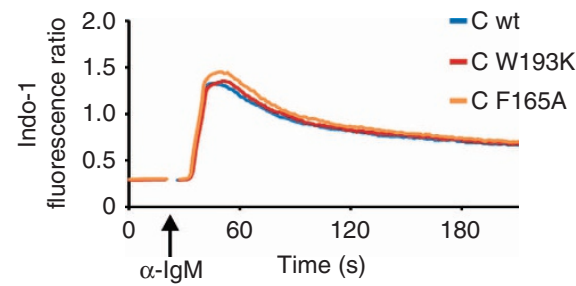

f

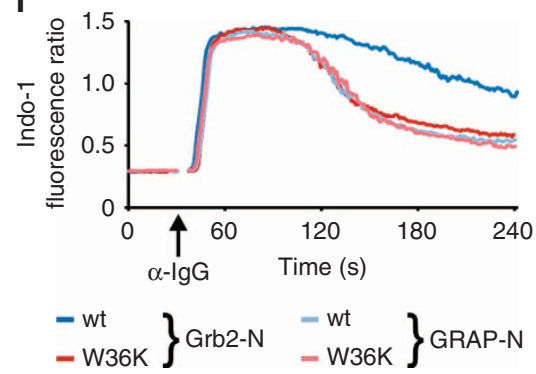

Figure 5 | The $\mathbf{N}$-terminal SH3 domain of Grb2 mediates enhanced $\mathbf{C a}^{2}+$ signalling downstream of the ITT. (a) Schematic illustration of chimeric receptor variants used in this study. Chimeric constructs consisting of mouse YF-mutant $\gamma 2$ am fused to the N-terminal (b) or C-terminal (c) SH3 domains of Grb2 were retrovirally expressed in DG75 B cells. The SH3 domains were either wild type (wt, blue curves) or inactivated by tryptophan to lysine (W36K and W193K, respectively, red curves) or a phenylalanine to alanine (F165A, orange curve) mutations. Cells were either stimulated with anti-lgG or anti-lgM F(ab') $)_{2}$ fragments (indicated by arrows) and $\mathrm{Ca}^{2}+$ mobilization was recorded in the presence of $1 \mathrm{mM}$ extracellular $\mathrm{CaCl}_{2}$. (d) $\mathrm{Chimeric}$ r2am-YF-chimeras containing the N-terminal SH3 domain of either Grb2 (wt: dark blue line, W36K: dark red line) or GRAP (wt: light blue line, W36K: light red line) were retrovirally expressed in murine splenic B cells and analysed as before. Data are representative of four independent experiments.

mIgG1-positive memory B-cell population. Subsequently, gBspecific IgG1 + memory B cells were isolated, transferred into Rag1-deficient recipient mice and challenged 6 days later with gB-containing virus-like particles (VLPs) (Fig. 4a). The usage of Rag1-deficient recipients allowed us to study the reactivation of the transferred memory B cells in the absence of T-cell help. The gB-specific IgG1 responses were determined for 20 days, which revealed that wild-type memory $B$ cells gave rise to up to ninefold higher IgG1 titres as compared with Grb2-deficient cells (Fig. 4b). This difference was furthermore reflected by a substantially higher number of IgG1-secreting plasma cells in the spleens of the recipient mice 43 days after the challenge (Fig. $4 \mathrm{c}$ ). Similar results were obtained when Grb2 was deleted in all B cells using mb1-cre deleter mice (Supplementary Fig. 6). Taken together, our results show that Grb2 is essential for enhanced signalling of ITT-containing BCR isotypes, which greatly improves their $\mathrm{T}$-cell-independent reactivation on antigen reencounter in vivo.

The N-terminal Grb2 SH3 domain mediates ITT signalling. The adaptor protein Grb2 is composed of a central SH2 domain that is flanked by an $\mathrm{N}$-terminal and a C-terminal SH3 domain. Both $\mathrm{SH} 3$ domains were reported to have exclusive and overlapping interaction partners ${ }^{30}$. To investigate the role of the individual Grb2 SH3 domains in an mIgG-BCR-specific manner, we generated chimeric mIgG-molecules in which we fused the respective Grb2 $\mathrm{SH} 3$ domains to the cytoplasmic tail of YF-mutant $\gamma 2$ am (see Fig. 5a). As controls we used inactivated variants of these domains (W36K, W193K or F165A, respectively). All constructs were expressed in similar amounts on the surface of the human B-cell line DG75 (data not shown). Analysis of anti-IgG-induced $\mathrm{Ca}^{2}+$ mobilization showed that in the context of such chimeric BCRs the N-terminal (Fig. 5b) but not the C-terminal (Fig. 5c) SH3 domain of Grb2 was able to replace the ITT motif. As control, all cells were stimulated via their endogenous mIgM-BCRs to demonstrate equal $\mathrm{Ca}^{2+}$ mobilization capacities of the transductants (Fig. 5d,e).

To test whether the mIgG2a-Grb2N-SH3 chimera still required expression of endogenous Grb2, we expressed this receptor in our GRB2-deficient DG75 B cells. Clearly, the enhanced signalling capacity of the chimera did not require the presence of Grb2 (Supplementary Fig. 7), showing that selective incorporation of the N-terminal SH3 domain of Grb2 into the YF-mutant mIgG$\mathrm{BCR}$ complex was sufficient to restore the $\mathrm{Ca}^{2+}$-promoting effect of the ITT.

Besides Grb2 we identified the Grb2-related adaptor protein (GRAP) as a second direct ITT-binding protein (Supplementary Fig. 8). To test whether GRAP can fulfil a similar role in ITT signalling as Grb2, we generated chimeras of mIgG2a and the individual $\mathrm{SH} 3$ domains of GRAP. However, in contrast to the N-terminal SH3 domain of Grb2 both SH3 domains of GRAP failed to boost BCR-induced $\mathrm{Ca}^{2+}$ mobilization in the context of 

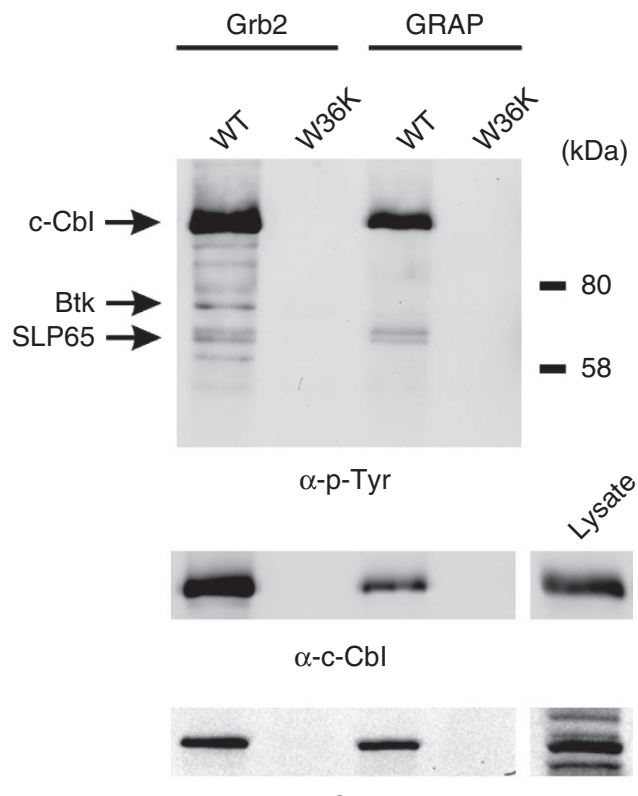

$\alpha-$ Sos

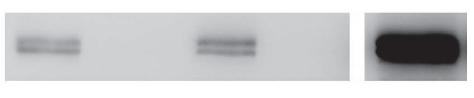

$\alpha-S L P 65$

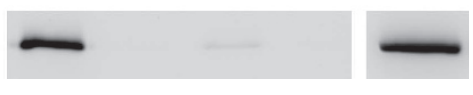

$\alpha-B t k$

Figure 6 | Interaction partners of the N-terminal Grb2 SH3 domain in B cells. DG75 B cells were stimulated via their BCR for 3 min to induce tyrosine phosphorylation of signalling proteins. Subsequently, lysates of these cells were used for affinity purifications with GST-coupled N-terminal SH3 domains of Grb2 and GRAP. Inactivated (W36K) variants were used as controls. Purified proteins were analysed by immunoblotting with antiphosphotyrosine ( $\alpha$-p-Tyr) antibodies (upper panel) and antibodies to c-Cbl, Sos, SLP65 and Btk as indicated. To assess the efficiency of affinity purification, signals of lysates (equivalent to $2 \%$ of input lysates) are shown on the right. Data are representative of four independent experiments.

chimeric mIgG2a-BCRs in primary mouse B cells (Fig. $5 \mathrm{f}$ and data not shown). These results show that the N-terminal $\mathrm{SH} 3$ domain of Grb2 specifically interacts with positive regulators of $\mathrm{Ca}^{2}+$ mobilization in B cells.

To identify candidate proteins that may mediate the enhanced $\mathrm{Ca}^{2+}$ signalling downstream of $\mathrm{Grb} 2$, we performed affinity purifications with the N-terminal $\mathrm{SH} 3$ domains of Grb2 and GRAP from lysates of BCR-activated human B cells and compared their interaction partners by immunoblot analyses (Fig. 6 and Supplementary Fig. 9). The most prominent tyrosinephosphorylated protein that was purified with both $\mathrm{SH} 3$ domains co-migrated with the c-Cbl (2nd panel from the top) and Cbl-b (not shown) ubiquitin ligases. Furthermore, both $\mathrm{SH} 3$ domains associated with the guanine nucleotide exchange factor Sos and weakly bound to SLP65. As the GRAP N-SH3 domain could not functionally replace the N-SH3 of Grb2 (see Fig. 5), these common interaction partners were most likely not involved in ITT-mediated $\mathrm{Ca}^{2}+$ signalling. In searching for specific interaction partners of the $\mathrm{N}-\mathrm{SH} 3$ of Grb2 that are known to regulate $\mathrm{Ca}^{2+}$ mobilization in B cells, we identified the PTK Btk as a novel interaction partner of Grb2 but not GRAP (Fig. 6, bottom panel). Noteworthy, we did not observe associations of the
N-terminal Grb2 SH3 domain with Src- or Syk-family PTKs nor with PLC- $\gamma$ isoforms (data not shown). Given the welldocumented role of Btk in BCR-induced $\mathrm{Ca}^{2+}$ mobilization, we investigated its putative function in ITT signalling in more detail.

Grb2 amplifies signalling of memory-type BCRs via Btk. To test whether recruitment of Btk to the mIgG-BCR represents an essential ITT effector mechanism, we generated a BTK-deficient variant of DG75 cells by homologous recombination (for details see Supplementary Fig. 10) and expressed the chimeric mIgG2aYF-Grb2-SH3 constructs in these BTK-deficient cells. Indeed, the signal-amplifying effect of the N-terminal Grb2 SH3 domain was abolished in the absence of Btk expression (Supplementary Fig. 11). Next, we retrovirally reconstituted the cells with a Citrine-tagged variant of wild-type Btk. This strategy gave rise to two populations of cells that were either Btk-negative (Fig. 7a, Cit-neg) or Btk-positive (Cit-pos) and hence could be differentiated during $\mathrm{Ca}^{2}+$ flux analysis by gating. In contrast to the situation in Citrine-positive cells (Fig. 7b, Cit-pos) and wild-type cells (see Fig. 5), the N-terminal SH3 domain of Grb2 did not amplify $\mathrm{Ca}^{2}+$ mobilization compared with the C-terminal Grb2 $\mathrm{SH} 3$ domain in the absence of Btk (Fig. 7b, Cit-neg). As control the different transfectants were stimulated via their endogenous mIgM-BCRs (Fig. 7c). To test whether a direct interaction between Grb2 and Btk was required for this $\mathrm{Ca}^{2+}$-promoting circuit, we mapped and inactivated the Grb2-binding site in Btk (data not shown). However, inactivation of the respective prolinerich motif in Btk caused an almost complete loss of function of Btk and hence did not provide conclusive evidence for the need of a direct interaction between the two proteins in this process (data not shown, manuscript in preparation). To circumvent this drawback, we mapped the amino-acid residues in the N-terminal $\mathrm{SH} 3$ domain of Grb2 that are necessary for its interaction with Btk by constructing a series of Grb2/GRAP chimeric SH3 domains (see Supplementary Figure 12). With this approach we identified three amino acids at the very N-terminus of the Grb2 $\mathrm{N}-\mathrm{SH} 3$ that restore binding to Btk if transplanted into the GRAP $\mathrm{N}-\mathrm{SH} 3$. The respective chimeric $\mathrm{SH} 3$ domain (referred to as 'XIII') was fused to ITT-mutant mIgG2a as before and transfected into DG75 B cells. Indeed, this Btk-binding variant of the GRAP $\mathrm{N}-\mathrm{SH} 3$ amplified BCR-induced $\mathrm{Ca}^{2}+$ mobilization as well as the Grb2 N-SH3 domain (Fig. 7d). However, when we expressed this chimeric mIgG-BCR in BTK-deficient cells, the $\mathrm{Ca}^{2+}$-amplifying effect of the XIII SH3 domain variant was abolished (Fig. 7e).

To test the signalling capacity of the intact ITT in the absence of Btk, we expressed wild type and ITT-mutant mIgG-BCRs in our BTK-deficient DG75 cells. In this experimental setting, the ITT in wild-type mIgG still evoked a moderately increased $\mathrm{Ca}^{2+}$ signal compared with ITT-mutant mIgG (Fig. 7f), suggesting that additional $\mathrm{Ca}^{2+}$-amplifying mechanisms operate in parallel to recruitment of Btk to ITT-bound Grb2.

ITT signalling increases the antigen sensitivity of mIgG-BCRs. Our data show that ITT/Grb2-mediated signal amplification has a pivotal function in reactivation of $\mathrm{mIgG-BCR}$-expressing memory $\mathrm{B}$ cells in vivo (see Fig. 4). To test whether this improved cellular activation is reflected by a heightened sensitivity of mIgG-BCRs compared with ITT-less BCR isotypes, we titrated stimulating anti-IgG $\mathrm{F}(\mathrm{ab})_{2}$ fragments and used the measurement of $\mathrm{Ca}^{2+}$ mobilization kinetics as a read-out for the effectiveness of BCR stimulation. The results showed that wild-type mIgG-BCRs needed $\sim 4$ times less stimulating agent to induce a $\mathrm{Ca}^{2}+$ peak comparable to that of ITT-mutant BCRs (Fig. 8a, compare green curve of wt mIgG $\left(2.5 \mu \mathrm{g} \mathrm{ml}^{-1}\right)$ with purple curve of YA-mutant 
a

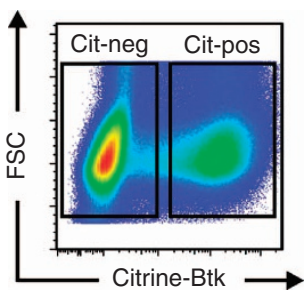

b

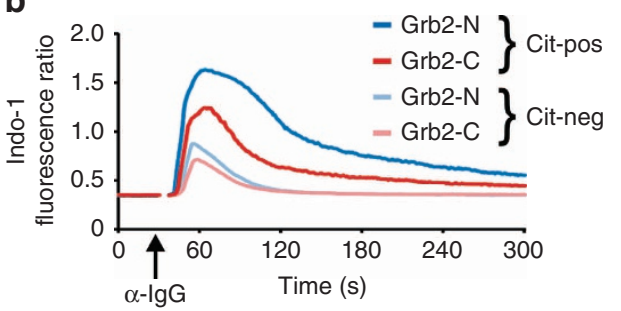

C

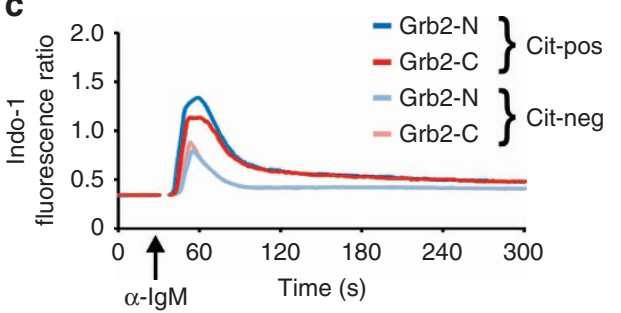

d

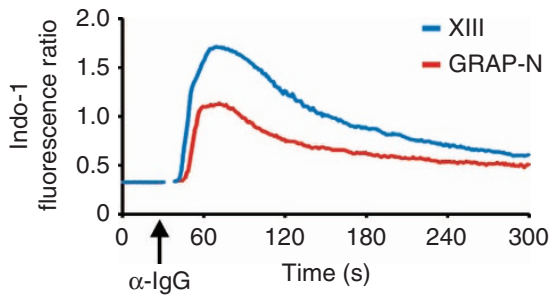

e

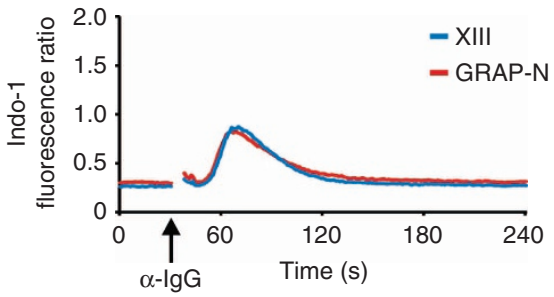

f

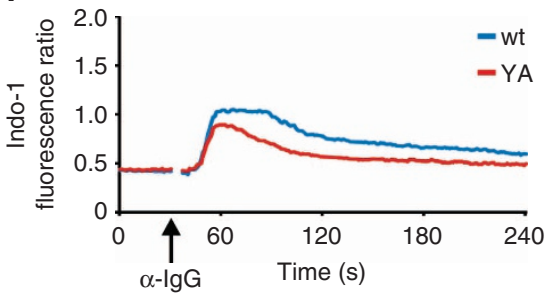

Figure 7 | Active amplification of $\mathbf{C a}^{\mathbf{2}}+$ signalling by $\mathbf{G r b 2}$ requires Btk. BTK-deficient DG75 B cells were retrovirally transduced to express chimeric $\gamma 2 \mathrm{am}$-YF molecules containing either the N-terminal (blue lines) or C-terminal (red lines) SH3 domains of Grb2. Subsequently, the cells were retrovirally transfected with Citrine-tagged Btk, resulting in two populations that were either Btk-negative (Cit-neg) or Btk-positive (Cit-pos) (a). BCR-induced Ca2+ mobilization was analysed in all populations on stimulation with polyclonal $F\left(a b^{\prime}\right)_{2}$ fragments to $\operatorname{lgG}(\mathbf{b})$ or $\operatorname{lgM}$ (c). (d) Wild-type DG75 cells expressing chimeric $\gamma 2$ am-YF molecules containing either the N-terminal SH3 domain of GRAP (red line) or a variant thereof (XIII) having three amino-acid substitutions at positions three, four and five (S3A, V4I and L6K, blue line) were analysed as before. (e) The same chimeric receptors as in (d) were analysed in BTK-deficient DG75 cells. (f) BTK-deficient DG75 cells were retrovirally transfected with wild type (wt, blue line) or tyrosine to alanine mutant (YA, red line) $\gamma 2$ am-encoding expression vectors and stimulated as before. Data are representative of at least three independent experiments.

mIgG $\left.\left(10 \mu \mathrm{g} \mathrm{ml}^{-1}\right)\right)$. Notably, stimulation with as little as $0.63 \mu \mathrm{g} \mathrm{ml}^{-1}$ anti-IgG $\mathrm{F}\left(\mathrm{ab}^{\prime}\right)_{2}$ fragments induced a longer lasting $\mathrm{Ca}^{2}+$ response in wild-type mIgG-expressing cells than optimal stimulation of ITT-mutant mIgG. Furthermore, we tested the activation of the Erk MAP kinase pathway in the cells under suboptimal stimulation conditions. In accordance with the enhanced $\mathrm{Ca}^{2+}$ flux, wild-type mIgG-BCRs induced a more robust and longer lasting phosphorylation of Erk proteins than ITT-mutant BCRs (Fig. 8b and Supplementary Fig. 13). Hence, signal amplification by the ITT/Grb2/Btk signalling circuit drastically lowers the amount of antigen needed to deliver an activation signal into the cell, which can explain why memory $\mathrm{B}$ cells with an intact ITT/Grb2 module have an advantage over cells that lack either component to get reactivated by small amounts of antigen.

\section{Discussion}

A hallmark of secondary immune responses is the switch in antibody isotype production from IgM to IgG. This observation goes back roughly half a century ${ }^{5}$ and has been refined more recently in elegant in vivo studies ${ }^{3,4}$. However, the molecular mechanisms that underlie the preferential production of IgG antibodies on antigen reencounter remain incompletely understood. Recently, we showed that the cytoplasmic domains of membrane-bound $\gamma$ and $\varepsilon$ Ig heavy chains contain a signal amplification motif that we termed the ITT $^{6}$. In addition to the ITT, a putative PDZ domain-recruiting peptide motif containing the amino-acid sequence SSVV has been suggested to contribute to the improved responsiveness of $\mathrm{mIgG}$-expressing memory $\mathrm{B}$ cells $^{23}$. When comparing the two motifs in our cellular model systems, the SSVV motif had little effect on signal initiation by mIgG-BCRs and its role for IgG responses in vivo remains to be seen. However, a recent report demonstrated that the SSVVbinding protein SAP97 is dispensable for primary and secondary antibody responses as both processes were normal in immunodeficient recipient mice that had been repopulated with Dlg-1- (SAP97)-deficient fetal liver cells and hence lacked SAP97 expression in $\mathrm{B}$ and $\mathrm{T}$ cells ${ }^{31}$.

The ITT resembles tyrosine-based signalling motifs found in several other immune receptors like DAP10 and CD28 that have either stimulatory or costimulatory activity ${ }^{32}$. The ITT-like motifs in these receptors are mainly targets of Src PTKs ${ }^{33,34}$. However, in $\mathrm{mIgG}$-BCRs the ITT is inevitably incorporated into the ITAMcontaining microenvironment of the $\operatorname{Ig} \alpha / \beta$ signalling device where it gets phosphorylated by-most likely ITAM-bound-Syk (see Fig. 9). ITT phosphorylation creates a binding site for $\mathrm{SH} 2$ domain-containing effector proteins that confer costimulatory activity to mIgG-BCRs. Although the mIgG-ITT has a YXNM consensus motif that-on paper-predisposes for binding to both Grb2 adaptors via YXN and p85 subunits of PI3K via YXXM, only Grb2 family adaptors could be detected as direct interaction 
a

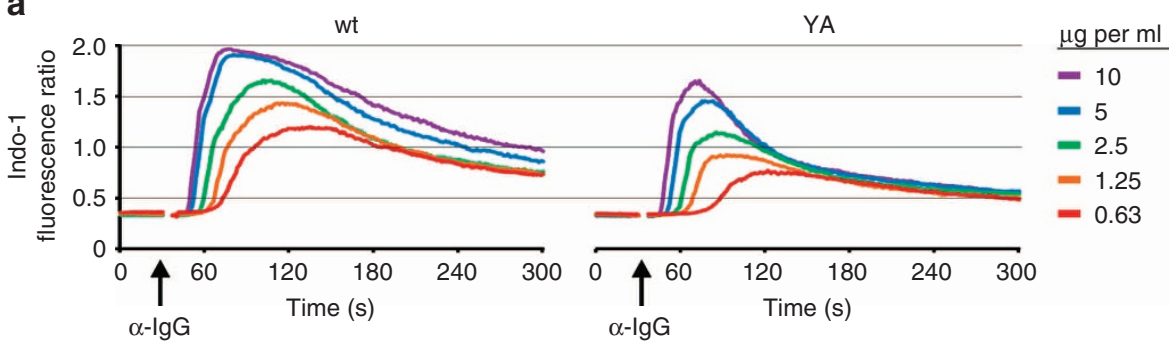

b

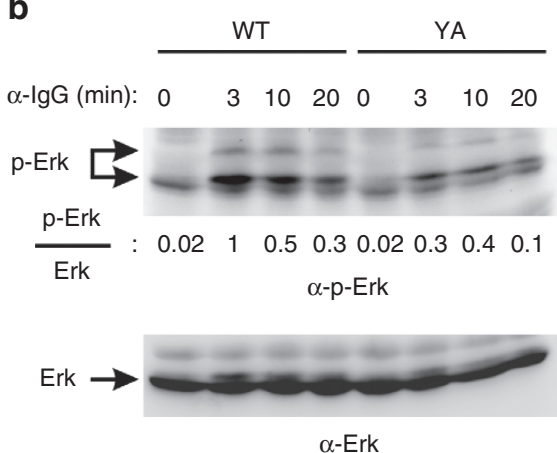

Figure 8 | The ITT increases the sensitivity of mlgG-BCRs for antigen. (a) $\mathrm{Ca}^{2}+$ mobilization kinetics of DG75 cells expressing wild type (wt) or ITT-mutant (YA) mlgG2a-BCRs on stimulation with decreasing amounts of anti-lgG $F\left(a b^{\prime}\right)_{2}$ fragments. Data are representative of two independent experiments. (b) The same cells were stimulated with $0.63 \mu \mathrm{g} \mathrm{ml}^{-1}$ anti-lgG $\mathrm{F}\left(a b^{\prime}\right)_{2}$ fragments for the indicated times, and activation of Erk was determined by immunoblot analysis with antibodies to phosphorylated Erk (upper panel). Anti-Erk served as a loading control (lower panel). The ratio of phosphorylated ( $p$-Erk) to total Erk is given. Data are representative of three independent experiments.

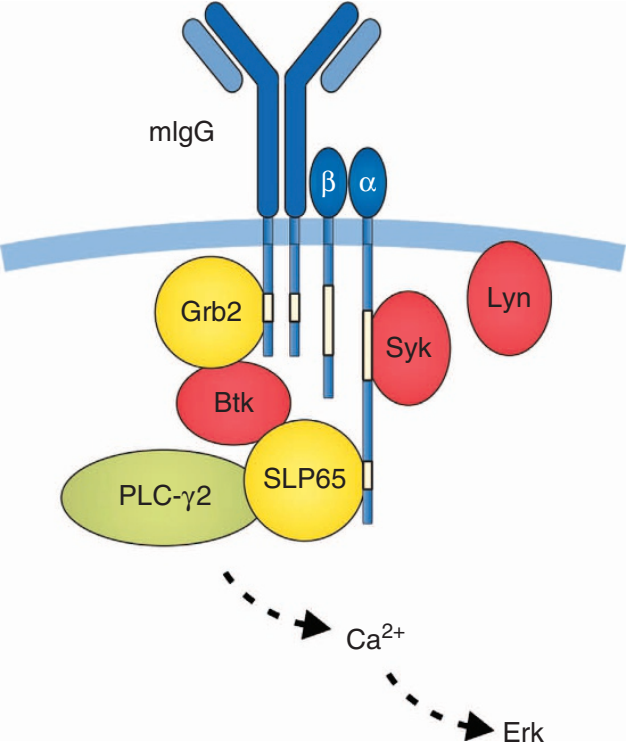

Figure 9 | Amplification of mlgG-BCR signalling by the ITT/Grb2/Btk signalling module. On phosphorylation by ITAM-bound Syk, the ITT motifs (white boxes in cytoplasmic mlgG tails) provide docking sites for the ubiquitous adaptor protein Grb2. Grb2 in turn brings along Bruton's tyrosine kinase (Btk) via a constitutive interaction that is mediated by the $\mathrm{N}$-terminal SH3 domain of Grb2. The incorporation of Grb2/Btk into the $\mathrm{BCR}$ signalosome stabilizes the $\mathrm{Ca}^{2}+$ initiation complex consisting of Btk, the adaptor protein SLP65 (which in addition interacts with a non-ITAM tyrosine-phosphorylation motif in $\lg \alpha$ ) and phospholipase C- $\gamma 2$ (PLC- $\gamma 2$ ) at the activated receptor, and thereby lowers the threshold for activation of PLC $-\gamma 2$ by Btk. This active signal amplification loop is complemented by a passive signal amplification that is brought about by sequestration of ITTbound Grb2 from negative regulators of $\mathrm{Ca}^{2}+$ mobilization such as $\mathrm{CD} 22$ and Dok-3. partners (ref. 6 and this study). In the present work, these biochemical findings are corroborated by genetic model systems showing that Grb2 is essential for enhanced signalling of mIgGBCRs as well as the efficient reactivation of IgG-switched memory B cells by virus-like particles and subsequent production of IgG antibodies in vivo. A key role for Grb2 recruitment is furthermore reflected by the strict evolutionary conservation of the YXN core (but not YXXM) in all known vertebrate ITTs. Together, the available data from biochemical and genetic in vitro and in vivo model systems substantiate the importance of the mIgG tail and its ITT-Grb2 signalling module for the vigorous nature of secondary IgG antibody responses. However, the role of Btk in that process appears less clear. Humans with BTK-deficiency have very low B-cell numbers and hence have very low antibody titres of all isotypes. Secondary antibody responses in mice with compromised Btk function are variously affected, which might be caused by the usage of different mouse strains as well as divergent immunization protocols ${ }^{35-37}$. Furthermore, in mice-as opposed to humans-the Btk-related PTK Tec can compensate for loss of Btk in B cells ${ }^{37}$. Hence, it appears possible that in mouse B cells Tec complements Btk in the ITT pathway, which would explain the moderately affected production of IgG antibodies in mice lacking functional Btk. Furthermore, even in our human B-cell system the ITT still provoked a moderately enhanced $\mathrm{Ca}^{2+}$ signal in the absence of Btk, showing that further mechanisms contribute to ITT-based signal amplification. Noteworthy, sequestration of Grb2 from negative regulators of $\mathrm{Ca}^{2+}$ mobilization in B cells like Dok-3 and CD22 has been suggested as a way to improve the activation of PLC- $\gamma$ and subsequent second messenger generation ${ }^{17,24}$. More specifically, Grb2 molecules that are hooked to phospho-ITTs are not available for association with phosphorylated negative regulators, resulting in a passive signal amplification that complements the active signal amplification by recruitment of Btk to memory-type BCRs. The importance of the $\mathrm{Ca}^{2+}$ 
initiation complex for IgG production and memory responses is furthermore reflected by the observation that maintenance and reactivation of IgG1-switched B cells require the expression of the Btk target PLC- $\gamma 2$ (ref. 38).

To what extent BCR signals are required or even sufficient to induce terminal plasma cell differentiation is incompletely understood. Studies with a TD antigen of varying affinity showed that strong antigen recognition favours plasma cell generation $^{39-41}$. As the strength of BCR signals rises with increasing affinity for antigen ${ }^{42}$, a picture emerges in which a $\mathrm{B}$ cell has two ways to increase its BCR signalling strength. First, by improving the affinity of its BCR for antigen and, second, by incorporating the signal-amplifying ITT into the BCR. Both modifications lead to enhanced intracellular $\mathrm{Ca}^{2}+$ mobilization, which seems to support the generation of plasma cells. Thus, B cells expressing ITT-containing BCRs have an advantage in contributing to antibody production over those cells expressing ITT-less BCRs if their antigen-affinities are the same. This may, at least in part, explain why IgG-switched memory B cells are preferentially reactivated by antigen over IgM-expressing memory cells ${ }^{4}$. However, additional mechanisms are likely to contribute to that process. For example, a recent study showed that certain transcription factors like Bach2 are downregulated specifically in mIgG1-expressing memory B cells, which may facilitate their rapid differentiation into plasma cells ${ }^{43}$.

In sum, class switching to IgG, IgE or IgY isotypes incorporates a signal-amplifying 'costimulatory' signalling device into memory-type BCRs that enhances the cell sensitivity for antigen and thus improves their competitiveness and reactivation in secondary immune reactions.

\section{Methods \\ Cells and transfections. The human Burkitt lymphoma line DG75 was purchased from the German Collection of Microorganisms and Cell Cultures (DSMZ, Braunschweig, Germany). The cells were stably transfected with the cDNA of murine cationic amino-acid transporter $1(s l c 7 a 1)$ to make them susceptible for infection with MMLV-based retrovirus particles and were selected with blasticidin $\mathrm{S}\left(10 \mu \mathrm{g} \mathrm{ml}^{-1}\right)$. The resultant DG75EB cells were used in all experiments. DG75 cells deficient for $B T K$ or GRB2, respectively, are described in detail in Supplementary Figs and were transfected wtih slc7al alike. Ramos cells expressing murine $\gamma 2$ am variants were previously described ${ }^{6}$. All cells were cultured in RPMI1640 + Glutamaxx (Biochrome) supplemented with $10 \%$ heat-inactivated FCS and antibiotics.}

Expression vectors. Retroviral expression vectors for mouse $\gamma 2 \mathrm{am}$ immunoglobulin heavy chains were previously described ${ }^{6}$. Mutations were introduced via PCR and verified by sequencing. Chimeric $\gamma 2 \mathrm{am}-\mathrm{SH} 3$ domain constructs were generated by fusing the coding sequences of the respective $\mathrm{SH} 3$ domains to YF-mutant $\gamma 2 \mathrm{am}$ cDNA. Citrine-tagged $\gamma 2$ am molecules were generated in the same way. $\gamma 2 \mathrm{am}$ and Citrine were separated by the 12 amino-acid linker GGGAASGGGAAS (singleletter code for amino acids). All constructs were cloned into pMSCVpuro (Invitrogen). The cDNA of mouse Grb2 was cloned into pMiRFP containing an IRES-tagRFP (Evrogen) element. The cDNA of human Btk was cloned into pMSCVpuro-Citrine-C1 resulting in N-terminally Citrine-tagged Btk. cDNAs encoding chimeric SH3 domains were either chemically synthesized (Eurofins MWG Operon) or generated by PCR.

Analysis of BCR-induced $\mathbf{C a}^{2}+$ mobilization. For analysis of intracellular $\mathrm{Ca}^{2+}$ concentrations, cells were loaded with $1 \mu \mathrm{M}$ Indo-1-AM (Molecular Probes) in RPMI containing $10 \%$ FCS and $0.015 \%$ pluronic acid (Molecular Probes) at $30^{\circ} \mathrm{C}$ for $30 \mathrm{~min}$ under mild agitation. After washing, cells were suspended in Krebs Ringer solution composed of $10 \mathrm{mM}$ HEPES ( $\mathrm{pH} 7.0$ ), $140 \mathrm{mM} \mathrm{NaCl}, 4 \mathrm{mM} \mathrm{KCl}$, $1 \mathrm{mM} \mathrm{MgCl} 2,1 \mathrm{mM} \mathrm{CaCl}$ and $10 \mathrm{mM}$ glucose. After monitoring basal $\mathrm{Ca}^{2+}$ concentrations for $30 \mathrm{~s}$, cells were stimulated with the indicated reagents. The fluorescence ratio of $\mathrm{Ca}^{2}+$ bound $(405 \mathrm{~nm})$ versus $\mathrm{Ca}^{2}+$ unbound Indo-1 $(530 \mathrm{~nm})$ was monitored on an LSR II cytometer (Becton Dickinson). Data analysis was done using FlowJo software (TreeStar).

Biochemical assays. For affinity purifications of mIgG2a-BCRs, $3 \times 10^{7}$ cells were stimulated with $10 \mu \mathrm{g} \mathrm{ml}^{-1}$ goat anti-mouse IgG antibodies (SouthernBiotech) for $5 \mathrm{~min}$ at $37^{\circ} \mathrm{C}$, then spun down and lysed in ice-cold lysis buffer containing $50 \mathrm{mM}$ Tris- $\mathrm{HCl}$ ( $\mathrm{pH} 7.8$ ), $137 \mathrm{mM} \mathrm{NaCl}, 0.5 \mathrm{mM}$ EDTA, $1 \mathrm{mM}$ sodium orthovanadate,
$10 \%(\mathrm{w} / \mathrm{v})$ glycerol, $1 \%(\mathrm{w} / \mathrm{v})$ NP40 supplemented with a protease inhibitor cocktail containing AEBSF, Aprotinin, Bestatin, E-64, Leupeptin and EDTA (Sigma Aldrich, P2714). Insoluble material was pelleted at $20.000 \times g$ for $10 \mathrm{~min}$ and cleared lysates were incubated with $30 \mu \mathrm{l}$ of protein A/G PLUS agarose slurry (Santa Cruz biotechnology) for $1 \mathrm{~h}$ at $4{ }^{\circ} \mathrm{C}$ with gentle agitation. After extensive washing with lysis buffer, bound proteins were released by boiling with Laemmli buffer and separated on $10 \%$ polyacrylamide gels. Tyrosine kinase inhibitors PP1, PP2 and BAY61-3606 were from Calbiochem and were used at a final concentration of $30 \mu \mathrm{M}$. Affinity purifications with GST fusion proteins were done as described for purification of mIgG-BCRs, except that $\sim 20 \mu \mathrm{g}$ of fusion proteins bound to glutathione-sepharose beads were used. Antibodies to $\beta$-Actin (clone 13E5), Btk (D3H5), phospho-BLNK-Y96, phospho-Erk1/2 (E10) and phosphotyrosine (100) were from Cell Signaling. Anti-pan-Erk (clone 16), anti-Grb2 (clone 81) and anti-c-Cbl (clone 17) were from BD Transduction Laboratories. Anti-GRAP was from Abnova, anti-SLP65 from BAbCo and anti-Syk (4D10) from Santa Cruz. All antibodies were used at 1:1,000 dilutions for western blotting. Quantification of western blot band intensities was done using Gel-Pro Analyser software (Media Cybernetics). Polyclonal goat $\mathrm{F}\left(\mathrm{ab}^{\prime}\right)_{2}$ fragments for BCR stimulation were from Jackson ImmunoResearch.

Mice. Grb2 $2^{f l / f l}$ mice were described previously ${ }^{26}$. Grb2 $2^{f l / f l} m b 1^{c r e /+}$ are on a mixed $\mathrm{BALB} / \mathrm{c} / \mathrm{C} 57 \mathrm{BL} / 6$ background. The $\mathrm{Grb} 2^{f l / f l} \mathrm{C} \gamma 1^{\mathrm{cre} /+}$ were backcrossed for ten generations to $\mathrm{C} 57 \mathrm{BL} / 6$ background. The $\mathrm{C} \gamma 1 \mathrm{cre}$ mice were a generous gift of $\mathrm{S}$. Casola ${ }^{29}$. Animal experiments were approved by a local ethics committee of the government of Central Franconia (Regierung Mittelfranken).

Retroviral transfections of primary mouse B cells. Splenocytes from 8- to 10-week-old female C57BL/6 mice (in house breeding) or corresponding B-cellspecific grb2-ko ( $\left(\mathrm{rb} 2^{f l / f l} \mathrm{mb1}^{\mathrm{cre} /+}\right)$ mice $^{26}$ were depleted of CD43-positive cells using anti-CD43 magnetic microbeads (Miltenyi Biotec). The remaining cells, which were $>90 \%$ positive for CD19 and IgM, were incubated for $24 \mathrm{~h}$ with $10 \mu \mathrm{g}$ per ml LPS (Sigma) in RPMI1640 + Glutamaxx supplemented with 10\% heatinactivated FCS and antibiotics. Subsequently $2 \times 10^{6}$ cells were infected with retrovirus-containing supernatants from Plat-E packaging cells supplemented with $3 \mu \mathrm{g} \mathrm{ml}^{-1}$ polybrene. Cells were used for assays 24 to $48 \mathrm{~h}$ after infection.

Adoptive cell transfer. For memory cell transfer, 2- to 4-month- old male and female $G r b 2^{\mathrm{fl} / \mathrm{fl}} m b 1^{\mathrm{cre} /+}$ and corresponding $G r b 2^{\mathrm{fl} / \mathrm{fl}} \mathrm{mb1} 1^{+I+}$ mice were immunized three times with the indicated amounts of hCMV glycoprotein B ( $g B$, a gift from Sanofi Pasteur, Lyon, France). Eighty-three days after the last immunization, splenic cells were stained with B220-PE (RA3-6B2), IgG1-FITC (A85-1), IgG2a-FITC (R19-15), IgG2b-FITC (R12-3). B220 +, IgG1 + /IgG2a + /IgG2b + cells were sorted, additionally stained with gB-Cy5 and sorted again for B220 +, $\operatorname{IgG1}+/ \operatorname{IgG} 2 \mathrm{a}+/ \operatorname{IgG} 2 \mathrm{~b}+, \mathrm{gB}+$ cells. Cells were sorted directly into tubes with Rag1 $^{-/}$splenic cells (as filler cells). Subsequently, the cell suspensions containing equal numbers of memory B cells for each genotype were adoptively transferred into 6-month-old male and female NK-cell depleted (depletion with anti-NK1.1 Ab from BioXcell) Rag1 ${ }^{-1}$ recipient mice (in house breeding) via tail vein injection. NK-cell depletion is necessary because of the mixed background of Grb2 fl/fl $m b 1^{c r e l+}$ and $G r b 2^{f l f l} m b 1^{+/+}$mice. After 6 days recipient mice were challenged by intravenous (i.v.) injection of $2 \mu \mathrm{g}$ VLPs containing $\mathrm{gB}$ in their envelope and bled regularly. For memory cell transfer from Grb2 fl/fl $C \gamma 1^{c r e /+}$ or $G r b 2^{w t / w t}$ $C \gamma 1^{\text {cre }}+$ mice, two to four months old male and female animals were immunized three times with gB. 70 days after the last immunization CD19+ spleen cells were purified by magnetic cell sorting. Aliquots of CD19 + cells were FACS analysed for B220, IgG1, IgG2a, IgG2b and gB. CD19 + cells corresponding to 1,000 B220 +, $\mathrm{IgG} 1+/ \mathrm{IgG} 2 \mathrm{a}+/ \mathrm{IgG} 2 \mathrm{~b}+, \mathrm{gB}+$ cells of each genotype were transferred i.v. into 2- to 4-month-old male and female Rag1 ${ }^{-/-}$mice. Six days later recipient mice were challenged by i.v. injection of $2 \mu \mathrm{g}$ VLPs and bled regularly.

ELISA. To measure the gB-specific serum IgG titres, MaxiSorp plates (Nunc) were coated with $0.5 \mu \mathrm{g} \mathrm{ml}^{-1}$ soluble glycoprotein $\mathrm{B}(\mathrm{gB})$ of hCMV in $15 \mathrm{mM} \mathrm{Na}_{2} \mathrm{CO}_{3}$, $35 \mathrm{mM} \mathrm{NaHCO}_{3}$ (pH 9.6) overnight at $4{ }^{\circ} \mathrm{C}$. Blocking was performed with $5 \% \mathrm{FCS}$ $0.05 \%$ Tween 20 in PBS for at least $2 \mathrm{~h}$ at $37^{\circ} \mathrm{C}$ or overnight at $4{ }^{\circ} \mathrm{C}$. Plates were incubated with serially diluted serum overnight at $4^{\circ} \mathrm{C}$. Mixed sera of late immunization time points were used as a standard. gB-specific total IgG or IgG1 antibodies were detected by alkaline phosphatase-linked anti-IgG or anti-IgG1 antibodies (Southern Biotechnology), which were incubated on the plates for $2 \mathrm{~h}$ at $37^{\circ} \mathrm{C}$.

ELISPOT. Coating and blocking of plastic plates was done as described for ELISA plates. Cells were serially diluted ranging from $2 \times 10^{6}$ per well to about 7,800 cells per well. Following incubation overnight at $37^{\circ} \mathrm{C}$, cells were discarded and plates were incubated with $1 \mu \mathrm{g} \mathrm{ml}^{-1}$ anti-IgG1-alkaline phosphatase in PBS with $1 \%$ gelatin, $1 \%$ Tween 20 for $1 \mathrm{~h}$ at $37^{\circ} \mathrm{C}$. 5-Bromo-4-chloro-3-indolylphosphate was used as substrate for detection. 


\section{References}

1. Yoshida, T. et al. Memory B and memory plasma cells. Immunol. Rev. 237, 117-139 (2010)

2. Tangye, S. G. \& Tarlinton, D. M. Memory B cells: effectors of long-lived immune responses. Eur. J. Immunol. 39, 2065-2075 (2009).

3. Dogan, I. et al. Multiple layers of B cell memory with different effector functions. Nat. Immunol. 10, 1292-1299 (2009).

4. Pape, K. A., Taylor, J. J., Maul, R. W., Gearhart, P. J. \& Jenkins, M. K. Different B cell populations mediate early and late memory during an endogenous immune response. Science 331, 1203-1207 (2011).

5. Uhr, J. W. The Heterogeneity of the Immune Response. Science 145, 457-464 (1964).

6. Engels, N. et al. Recruitment of the cytoplasmic adaptor Grb2 to surface IgG and IgE provides antigen receptor-intrinsic costimulation to class-switched B cells. Nat. Immunol. 10, 1018-1025 (2009).

7. Liu, W., Meckel, T., Tolar, P., Sohn, H. W. \& Pierce, S. K. Intrinsic properties of immunoglobulin IgG1 isotype-switched B cell receptors promote microclustering and the initiation of signaling. Immunity 32, 778-789 (2010).

8. Schamel, W. W. \& Reth, M. Monomeric and oligomeric complexes of the B cell antigen receptor. Immunity 13, 5-14 (2000).

9. Tolar, P., Sohn, H. W. \& Pierce, S. K. The initiation of antigen-induced B cell antigen receptor signaling viewed in living cells by fluorescence resonance energy transfer. Nat. Immunol. 6, 1168-1176 (2005).

10. Reth, M. Antigen receptor tail clue. Nature 338, 383-384 (1989).

11. Rolli, V. et al. Amplification of B cell antigen receptor signaling by a Syk/ITAM positive feedback loop. Mol. Cell 10, 1057-1069 (2002).

12. Engels, N., Wollscheid, B. \& Wienands, J. Association of SLP-65/BLNK with the B cell antigen receptor through a non-ITAM tyrosine of Ig-alpha. Eur. J. Immunol. 31, 2126-2134 (2001).

13. Kabak, S. et al. The direct recruitment of BLNK to immunoglobulin alpha couples the B-cell antigen receptor to distal signaling pathways. Mol. Cell Biol. 22, 2524-2535 (2002)

14. Patterson, H. C., Kraus, M., Kim, Y. M., Ploegh, H. \& Rajewsky, K. The B cell receptor promotes $\mathrm{B}$ cell activation and proliferation through a non-ITAM tyrosine in the Igalpha cytoplasmic domain. Immunity 25, 55-65 (2006).

15. Ishiai, M., Sugawara, H., Kurosaki, M. \& Kurosaki, T. Cutting edge: association of phospholipase C-gamma 2 Src homology 2 domains with BLNK is critical for B cell antigen receptor signaling. J. Immunol. 163, 1746-1749 (1999).

16. Su, Y. W. et al. Interaction of SLP adaptors with the SH2 domain of Tec family kinases. Eur. J. Immunol. 29, 3702-3711 (1999).

17. Stork, B. et al. Grb2 and the non-T cell activation linker NTAL constitute a $\mathrm{Ca}(2+)$-regulating signal circuit in B lymphocytes. Immunity 21, 681-691 (2004).

18. Engelke, M., Engels, N., Dittmann, K., Stork, B. \& Wienands, J. Ca(2+) signaling in antigen receptor-activated B lymphocytes. Immunol. Rev. 218, 235-246 (2007)

19. Dellis, O. et al. Ca2 + entry through plasma membrane IP3 receptors. Science 313, 229-233 (2006).

20. Kaisho, T., Schwenk, F. \& Rajewsky, K. The roles of gamma 1 heavy chain membrane expression and cytoplasmic tail in IgG1 responses. Science 276, 412-415 (1997).

21. Martin, S. W. \& Goodnow, C. C. Burst-enhancing role of the IgG membrane tail as a molecular determinant of memory. Nat. Immunol. 3, 182-188 (2002).

22. Waisman, A. et al. IgG1 B cell receptor signaling is inhibited by CD22 and promotes the development of B cells whose survival is less dependent on Ig alpha/beta. J. Exp. Med. 204, 747-758 (2007).

23. Liu, W. et al. The scaffolding protein synapse-associated protein 97 is required for enhanced signaling through isotype-switched IgG memory B cell receptors. Sci. Signal. 5, ra54 (2012).

24. Stork, B. et al. Subcellular localization of Grb2 by the adaptor protein Dok-3 restricts the intensity of $\mathrm{Ca} 2+$ signaling in B cells. Embo J. 26, 1140-1149 (2007).

25. Jang, I. K., Zhang, J. \& Gu, H. Grb2, a simple adapter with complex roles in lymphocyte development, function, and signaling. Immunol. Rev. 232, 150-159 (2009).

26. Ackermann, J. A., Radtke, D., Maurberger, A., Winkler, T. H. \& Nitschke, L. Grb2 regulates B-cell maturation, B-cell memory responses and inhibits B-cell $\mathrm{Ca}(2+)$ signalling. Embo J. 30, 1621-1633 (2011).

27. Otipoby, K. L., Draves, K. E. \& Clark, E. A. CD22 regulates B cell receptormediated signals via two domains that independently recruit Grb2 and SHP-1. J. Biol. Chem. 276, 44315-44322 (2001).

28. Jang, I. K. et al. Growth-factor receptor-bound protein-2 (Grb2) signaling in B cells controls lymphoid follicle organization and germinal center reaction. Proc. Natl Acad. Sci. USA 108, 7926-7931 (2011).
29. Casola, S. et al. Tracking germinal center B cells expressing germ-line immunoglobulin gammal transcripts by conditional gene targeting. Proc. Natl Acad. Sci. USA 103, 7396-7401 (2006).

30. Neumann, K., Oellerich, T., Urlaub, H. \& Wienands, J. The B-lymphoid Grb2 interaction code. Immunol. Rev. 232, 135-149 (2009).

31. Hawkins, E. D. et al. Regulation of asymmetric cell division and polarity by Scribble is not required for humoral immunity. Nat. Commun. 4, 1801 (2013).

32. Engels, N. \& Wienands, J. The signaling tool box for tyrosine-based costimulation of lymphocytes. Curr. Opin. Immunol. 23, 324-329 (2011).

33. Vivier, E., Nunes, J. A. \& Vely, F. Natural killer cell signaling pathways. Science 306, 1517-1519 (2004).

34. Raab, M. et al. p56Lck and p59Fyn regulate CD28 binding to phosphatidylinositol 3-kinase, growth factor receptor-bound protein GRB-2, and T cell-specific protein-tyrosine kinase ITK: implications for T-cell costimulation. Proc. Natl Acad. Sci. USA 92, 8891-8895 (1995).

35. Khan, W. N. et al. Defective B cell development and function in Btk-deficient mice. Immunity 3, 283-299 (1995).

36. Khan, A. Q., Sen, G., Guo, S., Witte, O. N. \& Snapper, C. M. Induction of in vivo antipolysaccharide immunoglobulin responses to intact Streptococcus pneumoniae is more heavily dependent on Btk-mediated B-cell receptor signaling than antiprotein responses. Infect. Immun. 74, 1419-1424 (2006).

37. Ellmeier, W. et al. Severe B cell deficiency in mice lacking the tec kinase family members Tec and Btk. J. Exp. Med. 192, 1611-1624 (2000).

38. Hikida, M. et al. PLC-gamma2 is essential for formation and maintenance of memory B cells. J. Exp. Med. 206, 681-689 (2009).

39. Paus, D. et al. Antigen recognition strength regulates the choice between extrafollicular plasma cell and germinal center B cell differentiation. J. Exp. Med. 203, 1081-1091 (2006).

40. Phan, T. G. et al. High affinity germinal center B cells are actively selected into the plasma cell compartment. J. Exp. Med. 203, 2419-2424 (2006).

41. Chan, T. D. et al. Antigen affinity controls rapid T-dependent antibody production by driving the expansion rather than the differentiation or extrafollicular migration of early plasmablasts. J. Immunol. 183, 3139-3149 (2009).

42. Liu, W., Meckel, T., Tolar, P., Sohn, H. W. \& Pierce, S. K. Antigen affinity discrimination is an intrinsic function of the B cell receptor. J. Exp. Med. 207, 1095-1111 (2010).

43. Kometani, K. et al. Repression of the transcription factor Bach2 contributes to predisposition of IgG1 memory B cells toward plasma cell differentiation. Immunity 39, 136-147 (2013).

\section{Acknowledgements}

We thank Ines Heine for excellent technical assistance. Our work was funded by the Deutsche Forschungsgemeinschaft through projects EN 834/1-1, EN 834/2-1 and the TRR 130, the State of Lower Saxony and the Volkswagen Foundation (grant VWZN2828) as well as an intramural grant to NE.

\section{Authors contributions}

N.E. designed the research, performed experiments, supervised the project and wrote the paper, L.M.K. designed and performed experiments and analysed data, W.S. generated and analysed BTK-deficient DG75 cells, K.V. performed experiments and analysed data J.L. generated GRB2-deficient DG75 cells, D.R. and T.W. did cell transfer experiments, L.N. provided B-cell-specific Grb2-KO mice and analysed data, J.W. analysed the data and wrote the paper.

\section{Additional information}

Supplementary Information accompanies this paper at http://www.nature.com/ naturecommunications

Competing financial interests: The authors declare no competing financial interests

Reprints and permission information is available online at http://npg.nature.com/ reprintsandpermissions/

How to cite this article: Engels, N. et al. The immunoglobulin tail tyrosine motif upgrades memory-type BCRs by incorporating a Grb2-Btk signalling module. Nat. Commun. 5:5456 doi: 10.1038/ncomms6456 (2014).

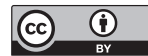

This work is licensed under a Creative Commons Attribution 4.0 International License. The images or other third party material in this article are included in the article's Creative Commons license, unless indicated otherwise in the credit line; if the material is not included under the Creative Commons license, users will need to obtain permission from the license holder to reproduce the material. To view a copy of this license, visit http://creativecommons.org/licenses/by/4.0/ 